\title{
ON THE EXTENSION OF WHITNEY ULTRAJETS OF BEURLING TYPE
}

\author{
ARMIN RAINER
}

\begin{abstract}
We prove a version of Whitney's extension theorem in the ultradifferentiable Beurling setting with controlled loss of regularity. As a byproduct we show the existence of continuous linear extension operators on certain spaces of Whitney ultrajets on arbitrary closed sets in $\mathbb{R}^{n}$.
\end{abstract}

\section{INTRODUCTION}

Ultradifferentiable versions of Whitney's extension theorem 28 seek a precise determination of how growth rates of the Whitney jets on closed subsets of $\mathbb{R}^{n}$ are preserved by their extensions to $\mathbb{R}^{n}$. There are different ways of defining these growth rates. In this paper they are measured in terms of a weight function $\omega$ in the framework of so-called Braun-Meise-Taylor classes. These classes of ultradifferentiable functions were introduced by Beurling [2] and Björck [3]. We shall work with the reformulation (and generalization) due to Braun, Meise, and Taylor [6]. With each weight function $\omega$ two types of Braun-Meise-Taylor classes of functions on $\mathbb{R}^{n}$ can be associated: the Beurling type $\mathcal{E}^{(\omega)}\left(\mathbb{R}^{n}\right)$ and the Roumieu type $\mathcal{E}^{\{\omega\}}\left(\mathbb{R}^{n}\right)$. Similarly, one has for any closed subset $A \subseteq \mathbb{R}^{n}$ the classes $\mathcal{E}^{(\omega)}(A)$ and $\mathcal{E}^{\{\omega\}}(A)$ of Whitney ultrajets on $A$. See Section 2 for precise definitions.

The jet mapping $j_{A}^{\infty}$ which sends a smooth function $f$ on $\mathbb{R}^{n}$ to the infinite jet $\left(\left.f^{(\alpha)}\right|_{A}\right)_{\alpha}$ consisting of its partial derivatives of all orders restricted to $A$ induces the mappings $j_{A}^{\infty}: \mathcal{E}^{(\omega)}\left(\mathbb{R}^{n}\right) \rightarrow \mathcal{E}^{(\omega)}(A)$ and $j_{A}^{\infty}: \mathcal{E}^{\{\omega\}}\left(\mathbb{R}^{n}\right) \rightarrow \mathcal{E}^{\{\omega\}}(A)$ (by restriction). It is natural to ask under which conditions these mappings are surjective. The answer was given by Bonet, Braun, Meise, and Taylor [4] (see also Abanin[1]) who fully characterized those weight functions $\omega$ which admit an extension theorem preserving the class: The following conditions are equivalent.

- $j_{A}^{\infty}: \mathcal{E}^{(\omega)}\left(\mathbb{R}^{n}\right) \rightarrow \mathcal{E}^{(\omega)}(A)$ is surjective for every closed $A \subseteq \mathbb{R}^{n}$.

- $j_{A}^{\infty}: \mathcal{E}^{\{\omega\}}\left(\mathbb{R}^{n}\right) \rightarrow \mathcal{E}^{\{\omega\}}(A)$ is surjective for every closed $A \subseteq \mathbb{R}^{n}$.

- $\omega$ is a strong weight function, i.e.,

$$
\exists C>0 \forall t>0: \int_{1}^{\infty} \frac{\omega(t u)}{u^{2}} d u \leq C \omega(t)+C .
$$

Many partial contributions by several authors led to this final answer.

Date: January 8, 2021.

2020 Mathematics Subject Classification. 26E10, 30D60, 46E10, 58C25 .

Key words and phrases. Whitney extension theorem in the ultradifferentiable setting, Beurling type classes, controlled loss of regularity, properties of weight functions.

Supported by the Austrian Science Fund (FWF) Project P 32905-N. 
For weight functions $\omega$ that are not strong one is interested in characterizing weight functions $\sigma$ such that

$$
j_{A}^{\infty}\left(\mathcal{E}^{(\omega)}\left(\mathbb{R}^{n}\right)\right) \supseteq \mathcal{E}^{(\sigma)}(A) \quad \text { and } \quad j_{A}^{\infty}\left(\mathcal{E}^{\{\omega\}}\left(\mathbb{R}^{n}\right)\right) \supseteq \mathcal{E}^{\{\sigma\}}(A),
$$

since the extension involves an unavoidable loss of regularity. This question was initiated by Ehrenpreis [8] and solved for the singleton $A=\{0\}$ by Bonet, Meise, and Taylor [5] and for compact convex sets $A$ with non-empty interior by Langenbruch [14]. In our recent papers [23, 24] we solved this problem in the Roumieu case for all compact sets $A$; see Theorem 1 below. The purpose of this note is to prove a similar result in the Beurling case.

We want to add that the analogous problems for ultradifferentiable classes defined by weight sequences have been solved by Chaumat and Chollet 7 for general $A$, by Langenbruch [14] for compact convex sets $A$ with non-empty interior, and by Petzsche [18] and Schmets and Valdivia [26] for the singleton.

1.1. Results. Let us first recall

Theorem 1 (23, 24]). Let $\omega$ be a non-quasianalytic concave weight function. Let $\sigma$ be a weight function satisfying $\sigma(t)=o(t)$ as $t \rightarrow \infty$. Then we have $j_{A}^{\infty}\left(\mathcal{E}^{\{\omega\}}\left(\mathbb{R}^{n}\right)\right) \supseteq \mathcal{E}^{\{\sigma\}}(A)$ for every closed $A \subseteq \mathbb{R}^{n}$ if and only if

$$
\exists C>0 \forall t>0: \int_{1}^{\infty} \frac{\omega(t u)}{u^{2}} d u \leq C \sigma(t)+C .
$$

Our goal is to prove a version of Theorem 1 in the Beurling case. We follow the standard strategy of reducing the Beurling to the Roumieu case. This means roughly speaking that for a pair $(\omega, \sigma)$ of suitable weight functions and for a Whitney ultrajet $F$ of class $\mathcal{E}^{(\sigma)}$ one tries to find a related pair $(\tilde{\omega}, \tilde{\sigma})$ of weight functions which satisfy the assumptions of Theorem 11 and such that the jet $F$ is also of Roumieu class $\mathcal{E}^{\{\tilde{\sigma}\}}$. Then by Theorem 1 there exists an $\mathcal{E}^{\{\tilde{\omega}\}}$-extension to $\mathbb{R}^{n}$ which also is of class $\mathcal{E}^{(\omega)}$ provided that $\omega(t)=o(\tilde{\omega}(t))$ as $t \rightarrow \infty$.

We could not preserve the natural condition $(\underline{S})$ in the reduction procedure. Instead we prove an extension theorem of Beurling type under a slightly stronger condition. The following is our main result:

Theorem 2. Let $\omega$ be a non-quasianalytic concave weight function. Let $\sigma$ be a weight function satisfying $\sigma(t)=o(t)$ as $t \rightarrow \infty$. Suppose that there exists $r \in(0,1)$ such that

$$
\exists C>0 \forall t>0: \int_{1}^{\infty} \frac{\omega(t u)}{u^{1+r}} d u \leq C \sigma(t)+C .
$$

Then for every closed $A \subseteq \mathbb{R}^{n}$ we have $j_{A}^{\infty}\left(\mathcal{E}^{(\omega)}\left(\mathbb{R}^{n}\right)\right) \supseteq \mathcal{E}^{(\sigma)}(A)$.

We will see in Proposition 7 that the existence of $r \in(0,1)$ such that $\left(S_{r}\right)$ holds is equivalent to a condition (namely (3.2) below) which is suited for the reduction (see Lemma 13) in the sense that it can be transfered from $(\omega, \sigma)$ to $(\tilde{\omega}, \tilde{\sigma})$.

Definition 3. We will say that an (ordered) pair of weight functions $(\omega, \sigma)$ is $r$ strong if $\left(\mathrm{S}_{r}\right)$ holds. The pair $(\omega, \sigma)$ is simply called strong if it is 1-strong. Note that the pair $(\omega, \omega)$ is strong if and only if $\omega$ is a strong weight function.

Note that the condition $\left(S_{r}\right)$ appears naturally in the framework of ultraholomorphic extension theorems (see [12] and Remark 12). 
If the weight functions $\omega$ and $\sigma$ are equivalent (i.e., generate the same function spaces), then the pair $(\omega, \sigma)$ is strong if and only if it is $r$-strong for some $r \in(0,1)$ and, additionally, the weight function $\omega$ is strong (see Lemma 8). In that case we recover the result stated in the introduction, since any strong weight function is equivalent to a concave one and always satisfies $\omega(t)=o(t)$ as $t \rightarrow \infty$. In general the condition $\left(\overline{S_{r}}\right)$ for some $r \in(0,1)$ is strictly stronger than $(\underline{S})$ (see Example 11). Moreover, for the singleton $K=\{0\}$, by $\left[5\right.$, and for compact convex sets $K \subseteq \mathbb{R}^{n}$ with non-empty interior, by [14], the pair $(\omega, \sigma)$ being strong is equivalent to the inclusion $j_{K}^{\infty}\left(\mathcal{E}^{(\omega)}\left(\mathbb{R}^{n}\right)\right) \supseteq \mathcal{E}^{(\sigma)}(K)$. So it remains an open question if in Theorem 2 the condition $\left(\underline{S_{r}}\right)$ can be replaced by $(\underline{S})$.

Remark 4. (1) In Theorem 1 and Theorem 2 we may assume that the ultradifferentiable extension to $\mathbb{R}^{n}$ of any ultrajet $F$ on $A$ is real analytic on $\mathbb{R}^{n} \backslash A$. This follows from a result of Schmets and Validivia [25. It can also be seen easily by adapting the proof of Langenbruch [15, Theorem 13] which is based on a general approximation theorem of Whitney type developed in the same paper.

(2) Both Theorem 1(sufficiency of (S) ) and Theorem 2 follow from the respective results for compact sets $K \subseteq \mathbb{R}^{n}$, since both $\mathcal{E}^{\{\omega\}}$ and $\mathcal{E}^{(\omega)}$ admit partitions of unity if $\omega$ is non-quasianalytic. For the sake of completeness we give a short argument: Let $A \subseteq \mathbb{R}^{n}$ be closed and fix $F=\left(F^{\alpha}\right) \in \mathcal{E}^{[\omega]}(A)$; here $\mathcal{E}^{[\omega]}$ stands for $\mathcal{E}^{\{\omega\}}$ or $\mathcal{E}^{(\omega)}$. For $k \in \mathbb{N}_{\geq 1}$ consider the open annuli $U_{k}:=\left\{x \in \mathbb{R}^{n}: k-1<|x|<k+1\right\}$ and set $U_{0}:=\left\{x \in \mathbb{R}^{n}:|x|<1\right\}$. There exist functions $\varphi_{k} \in \mathcal{E}^{[\omega]}\left(\mathbb{R}^{n}\right)$ such that $0 \leq \varphi_{k} \leq 1, \operatorname{supp} \varphi_{k} \subseteq U_{k}$, and $\sum_{k=1}^{\infty} \varphi_{k}=1$. For each $k \in \mathbb{N}_{\geq 1}$ the jet $F_{k}:=\left(\left.F^{\alpha}\right|_{\bar{U}_{k}}\right)$ belongs to $\mathcal{E}^{[\omega]}\left(A \cap \bar{U}_{k}\right)$. So there exists $f_{k} \in \mathcal{E}^{[\omega]}\left(\mathbb{R}^{n}\right)$ such that $j_{A \cap \bar{U}_{k}}^{\infty}\left(f_{k}\right)=F_{k}$. Then $f:=\sum_{k=1}^{\infty} \varphi_{k} f_{k}$ is a function in $\mathcal{E}^{[\omega]}\left(\mathbb{R}^{n}\right)$ since on any compact set the sum is finite. Let $x \in A$ be fixed. Let $\ell$ be the unique integer such that $\ell-1 \leq|x|<\ell$. Then $\varphi_{k}=0$ near $x$ unless $k \in\{\ell, \ell+1\}$. For each $\alpha \in \mathbb{N}^{n}$

$$
\begin{aligned}
f^{(\alpha)}(x) & =\partial^{\alpha} \sum_{k=1}^{\infty} \varphi_{k}(x) f_{k}(x) \\
& =\sum_{\beta \leq \alpha}\left(\begin{array}{l}
\alpha \\
\beta
\end{array}\right) \varphi_{\ell}^{(\beta)}(x) f_{\ell}^{(\alpha-\beta)}(x)+\sum_{\beta \leq \alpha}\left(\begin{array}{l}
\alpha \\
\beta
\end{array}\right) \varphi_{\ell+1}^{(\beta)}(x) f_{\ell+1}^{(\alpha-\beta)}(x) \\
& =\sum_{\beta \leq \alpha}\left(\begin{array}{l}
\alpha \\
\beta
\end{array}\right) \partial^{\beta}\left(\varphi_{\ell}(x)+\varphi_{\ell+1}(x)\right) F^{\alpha-\beta}(x) \\
& =F^{\alpha}(x),
\end{aligned}
$$

since all summands with $|\beta|>0$ vanish.

(3) In the case that $K \subseteq \mathbb{R}^{n}$ is a compact set, for any Whitney ultrajet on $K$ the ultradifferentiable extension can be assumed to have compact support by the existence of suitable cut-off functions. In particular, the ultradifferentiable growth estimates are global. In [21, 23, 24, we did justice to this circumstance by writing $\mathcal{B}^{[\omega]}(K)$ for the space of Whitney ultrajets on $K$ and $\mathcal{B}^{[\omega]}\left(\mathbb{R}^{n}\right)$ for the space of ultradifferentiable functions on $\mathbb{R}^{n}$ of global class $\mathcal{B}^{[\omega]}$ (cf. Section 2). Clearly, $\mathcal{B}^{[\omega]}(K)=\mathcal{E}^{[\omega]}(K)$ and $\mathcal{B}^{[\omega]}\left(\mathbb{R}^{n}\right) \subsetneq \mathcal{E}^{[\omega]}\left(\mathbb{R}^{n}\right)$.

(4) In Theorem 1 Theorem 2 and also in Theorem 5 below one could remove the assumption that $\omega$ is non-quasianalytic, since the conditions $(\mathbf{S}),\left(\mathbf{S}_{r}\right)$ for some $r \in(0,1)$, and $j_{A}^{\infty}\left(\mathcal{E}^{[\omega]}\left(\mathbb{R}^{n}\right)\right) \supseteq \mathcal{E}^{[\sigma]}(A)$ all imply non-quasianalyticity of $\omega$. This is 
obvious for $(\underline{S})$ and hence also for $\left(\underline{S_{r}}\right)$ with $r \in(0,1)$, by Lemma 6 For the third condition it follows from [22].

As a by-product of the proofs of the Theorems 1 and 2 we show in Theorem 5 that, for $r$-strong pairs $(\omega, \sigma)$, for $r \in(0,1)$, of weight functions, there exists a continuous linear extension operator on suitable subspaces of $\mathcal{E}^{(\sigma)}(A)$ with values in $\mathcal{E}^{(\omega)}\left(\mathbb{R}^{n}\right)$, for every non-empty closed subset $A \subseteq \mathbb{R}^{n}$. By a continuous linear extension operator on a closed set $A \subseteq \mathbb{R}^{n}$ we mean any continuous linear rightinverse of $j_{A}^{\infty}$ with suitable domain and codomain.

Theorem 5. Let $\omega$ be a non-quasianalytic concave weight function. Let $\sigma$ be a weight function satisfying $\sigma(t)=o(t)$ as $t \rightarrow \infty$. Assume that $(\omega, \sigma)$ is r-strong for some $r \in(0,1)$. If $\tau$ is a weight function such that $\sigma(t)=o(\tau(t))$ as $t \rightarrow \infty$, then for each non-empty closed subset $A \subseteq \mathbb{R}^{n}$ there exists a continuous linear extension operator $T_{A}: \mathcal{E}^{\{\tau\}}(A) \rightarrow \mathcal{E}^{(\omega)}\left(\mathbb{R}^{n}\right)$.

A similar result has been obtained by Chaumat and Chollet [7, Theorem 31] in the setting of Denjoy-Carleman classes.

In the case that $\omega$ is a strong weight function it is well-understood when a continuous linear extension operator $\mathcal{E}^{(\omega)}(A) \rightarrow \mathcal{E}^{(\omega)}\left(\mathbb{R}^{n}\right)$ exists. If $A$ is the closure of a bounded domain with real analytic boundary, it always exists, and for the singleton $A=\{0\}$ it exists if and only if

$$
\forall C>0 \exists \delta>0 \exists R_{0} \geq 1 \forall R \geq R_{0}: \omega^{-1}(C R) \omega^{-1}(\delta R) \leq \omega^{-1}(R)^{2} ;
$$

by Meise and Taylor [17. Franken 9 proved that under this additional condition every closed set $A \subseteq \mathbb{R}^{n}$ has a continuous linear extension operator $\mathcal{E}^{(\omega)}(A) \rightarrow$ $\mathcal{E}^{(\omega)}\left(\mathbb{R}^{n}\right)$. In the Roumieu case extension operators $\mathcal{E}^{\{\omega\}}(A) \rightarrow \mathcal{E}^{\{\omega\}}\left(\mathbb{R}^{n}\right)$ do usually not exist; see Langenbruch 13 .

The proof of Theorem 2 is given in Section [5 it is based on the Reduction lemma 13 presented in Section 4 . Theorem 5 is proved in Section 6 .

Acknowledgement. The author wishes to thank David Nenning and Gerhard Schindl for helpful discussions and reading a preliminary version of the paper.

\section{Spaces of Ultradifferentiable functions AND Jets}

2.1. Weight functions. A weight function is any continuous increasing function $\omega:[0, \infty) \rightarrow[0, \infty)$ with $\omega(0)=0$ that satisfies

$$
\begin{aligned}
& \omega(2 t)=O(\omega(t)) \quad \text { as } t \rightarrow \infty, \\
& \log t=o(\omega(t)) \quad \text { as } t \rightarrow \infty, \\
& \varphi(t):=\omega\left(e^{t}\right) \text { is convex. }
\end{aligned}
$$

A weight function is called non-quasianalytic if

$$
\int_{1}^{\infty} \frac{\omega(t)}{t^{2}} d t<\infty
$$

Two weight functions $\omega$ and $\sigma$ are said to be equivalent if $\omega(t)=O(\sigma(t))$ and $\sigma(t)=O(\omega(t))$ as $t \rightarrow \infty$. For each weight function $\omega$ there is an equivalent weight function $\tilde{\omega}$ such that $\omega(t)=\tilde{\omega}(t)$ for large $t>0$ and $\left.\tilde{\omega}\right|_{[0,1]}=0$. It is thus no restriction to assume that $\left.\omega\right|_{[0,1]}=0$ when necessary. 
The Young conjugate $\varphi^{*}$ of $\varphi$ is defined by

$$
\varphi^{*}(t):=\sup _{s \geq 0}(s t-\varphi(s)), \quad t \geq 0 .
$$

Assuming $\left.\omega\right|_{[0,1]}=0$, we have that $\varphi^{*}$ is a convex increasing function satisfying $\varphi^{*}(0)=0, t / \varphi^{*}(t) \rightarrow 0$ as $t \rightarrow \infty$, and $\varphi^{* *}=\varphi$; cf. [6] and [4, Remark 1.2].

2.2. Function spaces. Let $\omega$ be a weight function, $U \subseteq \mathbb{R}^{n}$ open, and $\rho>0$. We consider the Banach space $\mathcal{E}_{\rho}^{\omega}(U):=\left\{f \in C^{\infty}\left(\mathbb{R}^{n}\right):\|f\|_{U, \rho}^{\omega}<\infty\right\}$, where

$$
\|f\|_{U, \rho}^{\omega}:=\sup _{x \in U, \alpha \in \mathbb{N}^{n}}\left|\partial^{\alpha} f(x)\right| \exp \left(-\frac{1}{\rho} \varphi^{*}(\rho|\alpha|)\right),
$$

and the locally convex spaces

$$
\begin{aligned}
& \mathcal{E}^{(\omega)}\left(\mathbb{R}^{n}\right):=\operatorname{proj}_{U \Subset \mathbb{R}^{n}} \operatorname{proj}_{k \in \mathbb{N}_{\geq 1}} \mathcal{E}_{1 / k}^{\omega}(U), \\
& \mathcal{E}^{\{\omega\}}\left(\mathbb{R}^{n}\right):=\operatorname{proj}_{U \Subset \mathbb{R}^{n}} \operatorname{ind}_{k \in \mathbb{N}_{\geq 1}} \mathcal{E}_{k}^{\omega}(U) .
\end{aligned}
$$

Then $\mathcal{E}^{(\omega)}\left(\mathbb{R}^{n}\right)$ and $\mathcal{E}^{\{\omega\}}\left(\mathbb{R}^{n}\right)$ are called Braun-Meise-Taylor classes of Beurling type and of Roumieu type, respectively.

Let $\omega$ and $\sigma$ be weight functions. We have the following inclusion relations:

$$
\begin{aligned}
\mathcal{E}^{(\omega)}\left(\mathbb{R}^{n}\right) \subseteq \mathcal{E}^{(\sigma)}\left(\mathbb{R}^{n}\right) & \Leftrightarrow \sigma(t)=O(\omega(t)) \text { as } t \rightarrow \infty, \\
\mathcal{E}^{\{\omega\}}\left(\mathbb{R}^{n}\right) \subseteq \mathcal{E}^{\{\sigma\}}\left(\mathbb{R}^{n}\right) & \Leftrightarrow \quad \sigma(t)=O(\omega(t)) \text { as } t \rightarrow \infty, \\
\mathcal{E}^{\{\omega\}}\left(\mathbb{R}^{n}\right) \subseteq \mathcal{E}^{(\sigma)}\left(\mathbb{R}^{n}\right) & \Leftrightarrow \quad \sigma(t)=o(\omega(t)) \text { as } t \rightarrow \infty,
\end{aligned}
$$

cf. 19, Corollary 5.17]; in particular, $\omega$ and $\sigma$ are equivalent if and only if $\mathcal{E}^{(\omega)}\left(\mathbb{R}^{n}\right)=\mathcal{E}^{(\sigma)}\left(\mathbb{R}^{n}\right)$, respectively $\mathcal{E}^{\{\omega\}}\left(\mathbb{R}^{n}\right)=\mathcal{E}^{\{\sigma\}}\left(\mathbb{R}^{n}\right)$. The spaces $\mathcal{E}^{(\omega)}\left(\mathbb{R}^{n}\right) \subseteq$ $\mathcal{E}^{\{\omega\}}\left(\mathbb{R}^{n}\right)$ contain non-trivial functions with compact support if and only if $\omega$ is non-quasianalytic (cf. [6] or [19]). Since $C^{\omega}\left(\mathbb{R}^{n}\right)=\mathcal{E}^{\{t\}}\left(\mathbb{R}^{n}\right)$, the space of real analytic function on $\mathbb{R}^{n}$ is contained in $\mathcal{E}^{\{\omega\}}\left(\mathbb{R}^{n}\right)$ if and only if $\omega(t)=O(t)$ as $t \rightarrow \infty$, and it is contained in $\mathcal{E}^{(\omega)}\left(\mathbb{R}^{n}\right)$ if and only if $\omega(t)=o(t)$ as $t \rightarrow \infty$.

2.3. Whitney ultrajets. Let $A \subseteq \mathbb{R}^{n}$ be a closed non-empty set. Let $\mathcal{E}(A)$ be the set of Whitney jets (of class $C^{\infty}$ ) on $A$, i.e., $F=\left(F^{\alpha}\right)_{\alpha \in \mathbb{N}^{n}} \in C^{0}(A, \mathbb{R})^{\mathbb{N}^{n}}$ belongs to $\mathcal{E}(A)$ if for all compact subsets $K \subseteq A$, all $p \in \mathbb{N}$, and all $|\alpha| \leq p$ we have

$$
\left(R_{x}^{p} F\right)^{\alpha}(y)=o\left(|y-x|^{p-|\alpha|}\right) \quad \text { as }|y-x| \rightarrow 0, x, y \in K,
$$

where

$$
\left(R_{x}^{p} F\right)^{\alpha}(y):=F^{\alpha}(y)-\sum_{|\beta| \leq p-|\alpha|} \frac{(y-x)^{\beta}}{\beta !} F^{\alpha+\beta}(x) .
$$

By Whitney's extension theorem [28, the mapping $j_{A}^{\infty}: C^{\infty}\left(\mathbb{R}^{n}\right) \rightarrow \mathcal{E}(A)$, which is well-defined by Taylor's theorem, is surjective.

Let $\omega$ be a weight function. A Whitney jet $F=\left(F^{\alpha}\right)_{\alpha \in \mathbb{N}^{n}} \in \mathcal{E}(A)$ is called a $\omega$-Whitney ultrajet of Beurling type on $A$ if for all compact subsets $K \subseteq A$ and all integers $m \geq 1$ we have

$$
\|F\|_{K, 1 / m}^{\omega}:=\sup _{x \in K} \sup _{\alpha \in \mathbb{N}^{n}}\left|F^{\alpha}(x)\right| \exp \left(-m \varphi^{*}\left(\frac{|\alpha|}{m}\right)\right)<\infty
$$


and

$$
|F|_{K, 1 / m}^{\omega}:=\sup _{\substack{x, y \in K \\ x \neq y}} \sup _{p \in \mathbb{N}|\alpha| \leq p}\left|\left(R_{x}^{p} F\right)^{\alpha}(y)\right| \frac{(p+1-|\alpha|) !}{|x-y|^{p+1-|\alpha|}} \exp \left(-m \varphi^{*}\left(\frac{p+1}{m}\right)\right)<\infty .
$$

We denote by $\mathcal{E}^{(\omega)}(A)$ the locally convex space of all $\omega$-Whitney ultrajets $F$ of Beurling type on $A$ equipped with the project limit topology with respect to the system of seminorms $\|F\|_{K, 1 / m}^{\omega}+|F|_{K, 1 / m}^{\omega}$.

The space of $\omega$-Whitney ultrajets of Roumieu type on $A$ is

$$
\mathcal{E}^{\{\omega\}}(A):=\left\{F \in \mathcal{E}(A): \forall K \Subset A \exists m \in \mathbb{N}_{\geq 1}:\|F\|_{K, m}^{\omega}+|F|_{K, m}^{\omega}<\infty\right\}
$$

supplied with its natural locally convex topology.

\section{The condition $\left(S_{r}\right)$}

In this section we discuss $r$-strong pairs of weight functions.

Lemma 6. Let $\omega$ and $\sigma$ be weight functions. If the pair $(\omega, \sigma)$ is $r$-strong for some $r \in(0,1)$, then it is s-strong for all $s \in[r, 1]$. In particular, the pair $(\omega, \sigma)$ is strong and we have $\omega(t)=O(\sigma(t))$ as $t \rightarrow \infty$.

Proof. The first part of the lemma is trivial, since

$$
\int_{1}^{\infty} \frac{\omega(u t)}{u^{1+s}} d u \leq \int_{1}^{\infty} \frac{\omega(u t)}{u^{1+r}} d u
$$

for all $s \in[r, 1]$. Since $\omega$ is increasing, we have $\omega(t)=\omega(t) \int_{1}^{\infty} \frac{d u}{u^{2}} \leq \int_{1}^{\infty} \frac{\omega(u t)}{u^{2}} d u$ which implies the supplement.

In the following proposition we discover a condition which is equivalent of the pair $(\omega, \sigma)$ being $r$-strong for some $r \in(0,1)$. This condition can be preserved in the reduction procedure (see Lemma 13).

Proposition 7. Let $\omega$ and $\sigma$ be weight functions. Then the pair $(\omega, \sigma)$ is $r$-strong for some $r \in(0,1)$ if and only if

$$
\exists C>0 \exists K>H>1 \exists t_{0} \geq 0 \forall t \geq t_{0} \forall j \in \mathbb{N}_{\geq 1}: \omega\left(K^{j} t\right) \leq C H^{j} \sigma(t) .
$$

Proof. If $\omega$ and $\sigma$ satisfy $\left[\overline{S_{r}}\right]$ with $r \in(0,1)$ and $K>1$ is arbitrary, then since $\omega$ is increasing, for all integers $j \geq 0$, we have

$$
\begin{aligned}
\frac{\omega\left(K^{j} t\right)}{K^{r j}} & \leq \sum_{i=0}^{\infty} \frac{\omega\left(K^{i} t\right)}{K^{r i}}=\frac{K^{1+r}}{K-1} \sum_{i=0}^{\infty} \frac{\omega\left(K^{i} t\right)}{K^{(1+r)(i+1)}}\left(K^{i+1}-K^{i}\right) \\
& \leq \frac{K^{1+r}}{K-1} \int_{1}^{\infty} \frac{\omega(t u)}{u^{1+r}} d u \leq C \frac{K^{1+r}}{K-1}(\sigma(t)+1) .
\end{aligned}
$$

This implies (3.2) with $H:=K^{r}$. 
Suppose that (3.2) is fulfilled. Then we have $H=K^{r_{0}}$ with $r_{0}:=\log H / \log K \in$ $(0,1)$. Let $r \in\left(r_{0}, 1\right)$. Then for sufficiently large $t$,

$$
\begin{aligned}
\int_{1}^{\infty} \frac{\omega(t u)}{u^{1+r}} d u & =\sum_{j=0}^{\infty} \int_{K^{j}}^{K^{j+1}} \frac{\omega(u t)}{u^{1+r}} d u \leq \sum_{j=0}^{\infty} \omega\left(K^{j+1} t\right) \int_{K^{j}}^{K^{j+1}} \frac{1}{u^{1+r}} d u \\
& \leq \sum_{j=0}^{\infty} C H^{j+1} \sigma(t) r^{-1}\left(K^{-r j}-K^{-r(j+1)}\right) \\
& =C r^{-1}\left(K^{r}-1\right) \sigma(t) \sum_{j=0}^{\infty}\left(K^{r_{0}-r}\right)^{j+1}=C_{1} \sigma(t),
\end{aligned}
$$

since $K^{r_{0}-r}<1$. Thus $(\omega, \sigma)$ is $r$-strong.

The following lemma shows that Theorem 2 generalizes the extension result stated in the introduction.

Lemma 8. Let $\omega$ be a weight function. The following conditions are equivalent:

(1) $\omega$ is strong.

(2) Condition (3.2) holds with $\sigma=\omega$ and $C=1$.

(3) $(\omega, \omega)$ is $r$-strong for some $r \in(0,1)$.

Proof. (1) $\Rightarrow(2)$ : It was shown in [16] that the $\omega$ being strong is equivalent to

$$
\exists K>1: \limsup _{t \rightarrow \infty} \frac{\omega(K t)}{\omega(t)}<K .
$$

That means there exist $\epsilon>0$ and $t_{0} \geq 0$ such that

$$
\frac{\omega(K t)}{\omega(t)} \leq K-\epsilon, \quad \text { for all } t \geq t_{0}
$$

Thus condition (3.2) with $C=1, H=K-\epsilon$, and $\sigma=\omega$ follows by iteration.

$(2) \Rightarrow(3)$ follows from Proposition 7 .

$(3) \Rightarrow(1)$ is a consequence of Lemma 6 .

Let us discuss the relevance of condition (3.2) (or equivalently $\left(\underline{\left.S_{r}\right)}\right.$ for some $r \in(0,1))$ for the extension problem. Suppose that $\omega$ is a weight function that is not strong and let $A \subseteq \mathbb{R}^{n}$ be a closed non-empty set. We consider $j_{A}^{\infty} \mathcal{E}^{(\omega)}\left(\mathbb{R}^{n}\right)$ and look for spaces of Whitney ultrajets $\mathcal{E}^{(\sigma)}(A)$ as large as possible and contained in $j_{A}^{\infty} \mathcal{E}^{(\omega)}\left(\mathbb{R}^{n}\right)$. If $\bar{\omega}$ is any strong weight function such that $\omega(t)=O(\bar{\omega}(t))$ as $t \rightarrow \infty$, then $\mathcal{E}^{(\bar{\omega})}(A) \subseteq j_{A}^{\infty} \mathcal{E}^{(\omega)}\left(\mathbb{R}^{n}\right)$, since $\mathcal{E}^{(\bar{\omega})}(A)=j_{A}^{\infty} \mathcal{E}^{(\bar{\omega})}\left(\mathbb{R}^{n}\right)$ and $\mathcal{E}^{(\bar{\omega})}\left(\mathbb{R}^{n}\right) \subseteq \mathcal{E}^{(\omega)}\left(\mathbb{R}^{n}\right)$. The following Proposition 9 and Theorem 2 show that for any such $\bar{\omega}$ there is a weight function $\sigma$ such that $\mathcal{E}^{(\bar{\omega})}(A) \subsetneq \mathcal{E}^{(\sigma)}(A) \subseteq j_{A}^{\infty} \mathcal{E}^{(\omega)}\left(\mathbb{R}^{n}\right)$ (independently of $A$ ).

Proposition 9. Let $\omega$ be a weight function that is not strong. For any strong weight function $\bar{\omega}$ with $\omega(t)=O(\bar{\omega}(t))$ as $t \rightarrow \infty$ there exists a weight function $\sigma$ such that the pair $(\omega, \sigma)$ is $r$-strong for some $r \in(0,1)$ and we have $\sigma(t)=O(\bar{\omega}(t))$, $\bar{\omega}(t) \neq O(\sigma(t))$, and $\sigma(t)=o(t)$ as $t \rightarrow \infty$.

Proof. By Lemma 8 there exist constants $r_{0} \in(0,1), C>0$, and $t_{0} \geq 0$ such that

$$
\int_{1}^{\infty} \frac{\bar{\omega}(t u)}{u^{1+r_{0}}} d u \leq C \bar{\omega}(t) \quad \text { for all } t \geq t_{0} .
$$


Since $\omega(t)=O(\bar{\omega}(t))$ as $t \rightarrow \infty$, there is a constant $C_{1}>0$ and $t_{1} \geq t_{0}$ such that $\omega(t) \leq C_{1} \bar{\omega}(t)$ for all $t \geq t_{1}$.

Let $r \in\left(r_{0}, 1\right)$ and consider

$$
\sigma_{r}(t):=\int_{1}^{\infty} \frac{\omega(t u)}{u^{1+r}} d u, \quad t \geq 0 .
$$

The integral converges and we have $\sigma_{r}(t) \leq C_{2} \bar{\omega}(t)$ for all $t \geq t_{1}$, by (3.4). Moreover, $\sigma_{r}(t) \leq \sigma_{s}(t)$ for all $t \geq t_{1}$ provided that $r_{0}<s<r<1$. Since $\omega$ is increasing we have $\omega(t) \leq r \sigma_{r}(t) \leq \sigma_{r}(t)$ for all $r$ and all $t$.

Clearly, the pair $\left(\omega, \sigma_{r}\right)$ is $r$-strong for all $r \in\left(r_{0}, 1\right)$. By the inequalities $\omega(t) \leq$ $\sigma_{r}(t) \leq C_{2} \bar{\omega}(t)$ for all $t \geq t_{1}$ we may conclude that $\sigma_{r}(t)=o(t)$ as $t \rightarrow \infty$, since every strong weight function satisfies this, and $\log (t)=o\left(\sigma_{r}(t)\right)$ as $t \rightarrow \infty$, since $\omega$ has this property (cf. (2.2)). There is a constant $C_{3}>0$ such that $\sigma_{r}(2 t) \leq C_{3} \sigma_{r}(t)$ for large $t$ since $\omega$ has this property (cf. (2.1)). Since $t \mapsto \omega\left(e^{t}\right)$ is convex (cf. (2.3) ), it follows that also $t \mapsto \sigma_{r}\left(e^{t}\right)$ is convex for all $r$. Consequently, $\sigma_{r}$ is continuous and it is increasing since $\omega$ is increasing. So $\sigma_{r}$ is a weight function in the sense of Section 2.1

The computation (3.3) shows that for all $r \in(0,1)$ and all $t \geq 0$,

$$
\tau_{r}(t):=\sup _{j \in \mathbb{N}} \frac{\omega\left(K^{j} t\right)}{K^{r j}} \leq C_{4} \sigma_{r}(t) .
$$

On the other hand, for $0<s<r$,

$$
\begin{aligned}
\sigma_{r}(t) & =\int_{1}^{\infty} \frac{\omega(u t)}{u^{1+r}} d u=\sum_{j=0}^{\infty} \int_{K^{j}}^{K^{j+1}} \frac{\omega(u t)}{u^{1+r}} d u \\
& \leq \sum_{j=0}^{\infty} \frac{\omega\left(K^{j+1} t\right)}{K^{j(1+r)}}\left(K^{j+1}-K^{j}\right)=K^{r}(K-1) \sum_{j=0}^{\infty} \frac{\omega\left(K^{j+1} t\right)}{K^{s(j+1)}} \frac{K^{s(j+1)}}{K^{r(j+1)}} \\
& \leq K^{r}(K-1) \sum_{j=0}^{\infty}\left(K^{s-r}\right)^{j+1} \cdot \tau_{s}(t)=C_{5} \tau_{s}(t)
\end{aligned}
$$

for all $t \geq 0$. Arguing similarly as for $\sigma_{r}$ one sees easily that $\tau_{r}$, for $r \in\left(r_{0}, 1\right)$, form weight functions satisfying $\tau_{r}(t)=O(\bar{\omega}(t))$ and $\tau_{r}(t)=o(t)$ as $t \rightarrow \infty$. Moreover, $\tau_{r}$ satisfies (3.2) relative to $\omega$ by definition. We will use the interlacing properties (3.5) and (3.6) in order to show that $\bar{\omega}(t) \neq O\left(\sigma_{r}(t)\right)$ as $t \rightarrow \infty$ for all $r \in\left(r_{0}, 1\right)$. In fact, we will show that

$$
\tau_{s}(t) \neq O\left(\tau_{r}(t)\right), \quad \text { if } s<r,
$$

which implies $\sigma_{s}(t) \neq O\left(\sigma_{r}(t)\right)$ as $t \rightarrow \infty$, by (3.5) and (3.6). Consequently, for given $r \in\left(r_{0}, 1\right)$ take $s \in\left(r_{0}, r\right)$ and we see that $\sigma_{s}(t)=O(\bar{\omega}(t))$ and $\sigma_{s}(t) \neq$ $O\left(\sigma_{r}(t)\right)$ as $t \rightarrow \infty$ imply that $\bar{\omega}(t) \neq O\left(\sigma_{r}(t)\right)$ as $t \rightarrow \infty$.

Let us prove (3.7). Two cases may occur: First, if there exists $N \in \mathbb{N}$ and $T>0$ such that for all $t \geq T$

$$
\sup _{j \in \mathbb{N}} \frac{\omega\left(K^{j} t\right)}{K^{s j}}=\max _{0 \leq j \leq N} \frac{\omega\left(K^{j} t\right)}{K^{s j}},
$$

then $\tau_{s}(t) \leq \omega\left(K^{N} t\right)$ for such $t$ and since $\omega(2 t)=O(\omega(t))$ as $t \rightarrow \infty$ (cf. (2.1)), we may conclude that the weight functions $\omega$ and $\tau_{s}$ are equivalent. As a consequence 
$\omega$ is a strong weight function (by Lemma 6) contrary to the assumption of the proposition. Otherwise there are sequences $j_{n} \rightarrow \infty$ and $t_{n} \rightarrow \infty$ such that

$$
\frac{\omega\left(K^{j_{n}} t_{n}\right)}{K^{r j_{n}}} \geq \sup _{j \in \mathbb{N}} \frac{\omega\left(K^{j} t_{n}\right)}{K^{r j}}-\frac{1}{n}=\tau_{r}\left(t_{n}\right)-\frac{1}{n} .
$$

Consequently,

$$
\tau_{s}\left(t_{n}\right) \geq \frac{\omega\left(K^{j_{n}} t_{n}\right)}{K^{s j_{n}}}=K^{(r-s) j_{n}} \frac{\omega\left(K^{j_{n}} t_{n}\right)}{K^{r j_{n}}} \geq K^{(r-s) j_{n}}\left(\tau_{r}\left(t_{n}\right)-\frac{1}{n}\right) .
$$

This implies (3.7). The proof is complete.

Remark 10. The concave weight function $\kappa(t):=\int_{1}^{\infty} \frac{\omega(t y)}{y^{2}} d y$ (cf. [16, Remark 3.20] and [5, Proposition 1.3]) satisfies

$$
\kappa(t) \geq \frac{\omega\left(K^{j} t\right)}{K^{j}} \quad \text { for all } t>0, K>1, j \in \mathbb{N} .
$$

Indeed, this follows from $\omega \leq \kappa$ and concavity of $\kappa$ or more directly by

$$
\kappa(t)=\int_{1}^{\infty} \frac{\omega(t y)}{y^{2}} d y=\int_{K^{-j}}^{\infty} \frac{\omega\left(K^{j} t u\right)}{K^{j} u^{2}} d u \geq \frac{\omega\left(K^{j} t\right)}{K^{j}} \int_{1}^{\infty} \frac{1}{u^{2}} d u=\frac{\omega\left(K^{j} t\right)}{K^{j}},
$$

using that $\omega$ is increasing. Note that $\kappa$ defines the largest class of ultradifferentiable functions (respectively, jets) among all weight functions $\sigma$ such that $\int_{1}^{\infty} \frac{\omega(t y)}{y^{2}} d y=$ $O(\sigma(t))$ as $t \rightarrow \infty$ (i.e. such that the pair $(\omega, \sigma)$ is strong). In the situation of Proposition 9 we have $\omega(t) \leq \kappa(t) \leq C_{1} \sigma_{r}(t) \leq C_{2} \bar{\omega}(t)$ for sufficiently large $t$ and $r \in\left(r_{0}, 1\right)$ (thanks to (3.1)).

Example 11. For $\alpha>0$ we consider the weight functions $\omega_{\alpha}$ where

$$
\omega_{\alpha}(t)=\frac{t}{(\log t)^{\alpha}}, \quad \text { for sufficiently large } t .
$$

It is easy to see that the definition of $\omega_{\alpha}$ can be extended to small positive values of $t$ such that it is a weight function in the sense of Section 2.1. As observed in [5. Example 3.8] if $\alpha>1$ then

$$
\kappa_{\alpha}(t):=\int_{1}^{\infty} \frac{\omega_{\alpha}(t y)}{y^{2}} d y=\frac{\omega_{\alpha-1}(t)}{\alpha-1}, \quad \text { for large } t .
$$

(Apparently there is a small inaccuracy concerning the constant $\alpha-1$ in the above reference.) This shows that for $\alpha>1$ the pair $\left(\omega_{\alpha}, \omega_{\alpha-1}\right)$ is strong whereas the weight function $\omega_{\alpha}$ is not strong.

On the other hand the pair $\left(\omega_{\alpha}, \omega_{\alpha-1}\right)$ is not $r$-strong for any $r \in(0,1)$. Indeed it is easy to see that the condition (3.2) is violated. Otherwise there would exist constants $K>H>1$ such that for all integers $j \geq 1$ and sufficiently large $t$

$$
\frac{\omega_{\alpha}\left(K^{j} t\right)}{H^{j} \omega_{\alpha-1}(t)}=\left(\frac{K}{H}\right)^{j} \frac{(\log t)^{\alpha-1}}{(\log t+j \log K)^{\alpha}}
$$

is bounded which is obviously a contradiction. Note that

$$
\int_{1}^{\infty} \frac{\omega_{\alpha}(t y)}{y^{1+r}} d y=\omega_{\alpha-1}(t) \cdot t^{r-1} E_{\alpha}((r-1) \log t)
$$

where $E_{\alpha}(x):=\int_{1}^{\infty} y^{-\alpha} e^{-x y} d y$ is the exponential integral. 
Remark 12. The condition $\left(S_{r}\right)$ plays an interesting role in the context of ultraholomorphic sectorial extensions. The growth index $\gamma(\sigma, \omega)$ introduced in [12] is defined by

$$
\gamma(\sigma, \omega):=\sup \{s>0:(\omega, \sigma) \text { ist } 1 / s \text {-strong }\} .
$$

Note that a pair of weight functions $(\omega, \sigma)$ is $r$-strong for some $r \in(0,1)$ if and only if $\gamma(\sigma, \omega)>1$. The growth index for a pair of weight functions is a generalization of the growth index $\gamma(\omega):=\gamma(\omega, \omega)$ of a single weight function $\omega$ considered in 10, 11] imitating the growth index $\gamma(M)$ introduced by [27, for a weight sequence $M$. In the mentioned papers extension results of Borel-Ritt type are proved: given a formal power series with admissible growth behavior of the coefficients one looks for an ultraholomorphic function defined on a sector in the Riemann surface of the logarithm and asymptotic to the given series. The growth indices give sharp upper bounds for the aperture of the sectors on which the extension exists.

\section{The ReduCtion LEMMA}

The following lemma is the key to Theorem 2, Its proof contains ideas from 6 , Lemma 1.6, Lemma 1.7] and [4, Lemma 4.4].

Lemma 13 (Reduction lemma). Let $\omega$ and $\sigma$ be weight functions satisfying the following conditions:

(a) $\omega$ is concave.

(b) $\sigma(t)=o(t)$ as $t \rightarrow \infty$.

(c) $(\omega, \sigma)$ is $r$-strong for some $r \in(0,1)$.

Suppose that $f:[0, \infty) \rightarrow[0, \infty)$ is a function satisfying $\sigma(t)=o(f(t))$ as $t \rightarrow \infty$. Then there exist weight functions $\tilde{\omega}$ and $\tilde{\sigma}$ such that

$$
\omega(t)=o(\tilde{\omega}(t)), \sigma(t)=o(\tilde{\sigma}(t)) \text {, and } \tilde{\sigma}(t)=o(f(t)) \text { as } t \rightarrow \infty
$$

and with the following properties:

$(\tilde{a}) \tilde{\omega}$ is concave.

(尚) $\tilde{\sigma}(t)=o(t)$ as $t \rightarrow \infty$.

$(\tilde{c})(\tilde{\omega}, \tilde{\sigma})$ is $r$-strong for some $r \in(0,1)$.

Proof. We first claim that $\omega$ is of class $C^{1}$. Since $\omega$ is concave, for each $t>0$ the left-sided and the right-sided derivatives $\omega^{\prime-}(t)$ and $\omega^{\prime+}(t)$ exist and satisfy $\omega^{\prime+}(t) \leq \omega^{\prime-}(t)$. Since $\varphi=\omega \circ \exp$ is convex, we also have $\omega^{\prime-}(t) \leq \omega^{\prime+}(t)$. It follows that $\omega$ is differentiable. The monotonicity properties imply that $\omega$ is even of class $C^{1}$, indeed if $s \leq t$ then $\omega^{\prime}(t) \leq \omega^{\prime}(s)$ and $\omega^{\prime}(s) s \leq \omega^{\prime}(t) t$ and hence

$$
\omega^{\prime}(t) \leq \omega^{\prime}(s) \leq \omega^{\prime}(t) \frac{t}{s} \rightarrow \omega^{\prime}(t) \quad \text { as } t \rightarrow s .
$$

The condition $\omega(t)=o(t)$ as $t \rightarrow \infty$ implies that $\omega^{\prime}(t) \searrow 0$ as $t \rightarrow \infty$; indeed otherwise $\omega^{\prime}(t) \geq \epsilon>0$ for all $t$ as $\omega^{\prime}$ is decreasing and hence $\omega(t)=\int_{0}^{t} \omega^{\prime}(s) d s \geq \epsilon t$, a contradiction.

Auxiliary sequences. By Proposition 7 , assumption (c) implies that $\omega$ and $\sigma$ satisfy (3.2). So there exist constants $C>0, K>H>1$ and $t_{0} \geq 0$ such that

$$
\omega\left(K^{j} t\right) \leq C H^{j} \sigma(t), \quad \text { for all } t \geq t_{0}, j \geq 1 .
$$


Note that $\log (t)=o(\sigma(t))$ and $\sigma(t)=o(f(t))$ imply $f(t) \rightarrow \infty$ as $t \rightarrow \infty$. We define inductively three sequences $\left(x_{n}\right),\left(y_{n}\right)$, and $\left(z_{n}\right)$ with $x_{1}=y_{1}=z_{1}=0, x_{2}>0$, and the following properties:

$$
\begin{gathered}
x_{n}>\max \{2, K\} y_{n-1}+n, \\
\min \{t, f(t)\} \geq n^{2} \sigma(t), \quad \text { for all } t \geq x_{n}, \\
\omega\left(x_{n}\right) \geq 2^{n-i} \omega\left(z_{i}\right), \quad 1 \leq i \leq n-1, \\
\sigma\left(x_{n}\right) \geq 2^{n-i} \sigma\left(x_{i}\right), \quad 1 \leq i \leq n-1, \\
\omega^{\prime}\left(y_{n}\right)=\frac{n-1}{n} \omega^{\prime}\left(x_{n}\right), \\
\omega\left(z_{n}\right)=n \omega\left(y_{n}\right)-(n-1)\left(\omega\left(x_{n}\right)+\left(y_{n}-x_{n}\right) \omega^{\prime}\left(x_{n}\right)\right) .
\end{gathered}
$$

The point $y_{n}$ is well-defined by (4.6) since $\omega^{\prime}(t) \searrow 0$ as $t \rightarrow \infty$. The condition (4.7) is equivalent to

$$
\omega\left(y_{n}\right)-\omega\left(z_{n}\right)=(n-1)\left(\omega\left(x_{n}\right)+\left(y_{n}-x_{n}\right) \omega^{\prime}\left(x_{n}\right)-\omega\left(y_{n}\right)\right),
$$

that means the difference $\omega\left(y_{n}\right)-\omega\left(z_{n}\right)$ is the $(n-1)$-fold of the distance between the point with the abscissa $y_{n}$ on the tangent line at $x_{n}$ to the graph of $\omega$ and the point on the graph with the same abscissa. That $z_{n} \in\left[x_{n}, y_{n}\right]$ with this property exists follows from the assumption that $\omega$ is concave. Indeed, by continuity of $\omega$ it suffices to check that

$$
\omega\left(y_{n}\right)-\omega\left(x_{n}\right) \geq(n-1)\left(\omega\left(x_{n}\right)+\left(y_{n}-x_{n}\right) \omega^{\prime}\left(x_{n}\right)-\omega\left(y_{n}\right)\right)
$$

which is equivalent to

$$
\frac{\omega\left(y_{n}\right)-\omega\left(x_{n}\right)}{y_{n}-x_{n}} \geq \frac{n-1}{n} \omega^{\prime}\left(x_{n}\right) \stackrel{(4.6)}{=} \omega^{\prime}\left(y_{n}\right)
$$

and holds by concavity of $\omega$.

Definition of $\tilde{\omega}$. We define $\tilde{\omega}$ as follows:

$$
\tilde{\omega}(t):= \begin{cases}(n-1)\left(\omega\left(x_{n}\right)+\left(t-x_{n}\right) \omega^{\prime}\left(x_{n}\right)\right)-\sum_{i=1}^{n-2} \omega\left(z_{i+1}\right) & \text { if } x_{n} \leq t<y_{n}, \\ n \omega(t)-\sum_{i=1}^{n-1} \omega\left(z_{i+1}\right) & \text { if } y_{n} \leq t<x_{n+1} .\end{cases}
$$

Then $\tilde{\omega}$ is continuous (note that continuity at $y_{n}$ follows from (4.7)) and continuously differentiable (which follows from (4.6)). Since $\omega$ is increasing we may infer that $\tilde{\omega}$ is increasing. Being a $C^{1}$-function which piecewise is either affine-linear or concave $\tilde{\omega}$ must be concave. The same reasoning also shows that $t \mapsto \tilde{w}\left(e^{t}\right)$ is convex, since $\omega$ has this property.

Next we claim that

$$
\tilde{\omega}(t) \geq(n-2) \omega(t), \quad \text { if } t \in\left[x_{n}, x_{n+1}\right) \text { and } n \geq 2 .
$$




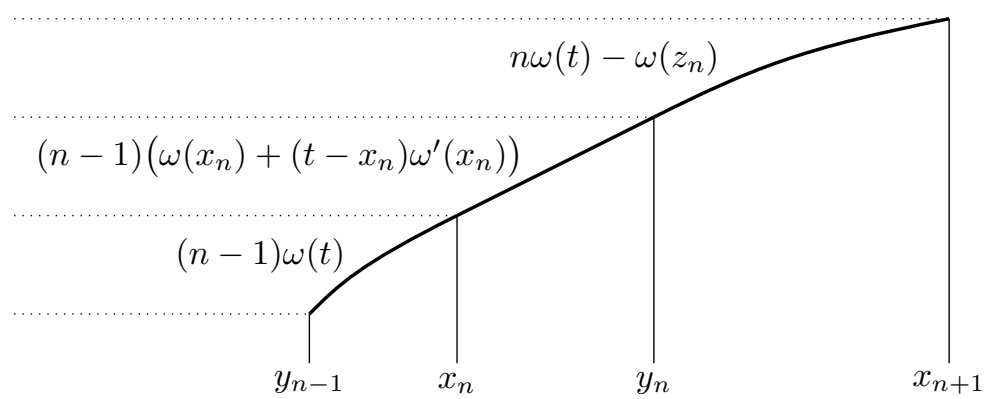

FiguRE 1. The graph of $\left[y_{n-1}, x_{n+1}\right] \ni t \mapsto \tilde{\omega}(t)+\sum_{i=1}^{n-2} \omega\left(z_{i+1}\right)$.

If $x_{n} \leq t \leq y_{n}$ then by concavity of $\omega$

$$
\begin{aligned}
\tilde{\omega}(t) & =(n-1)\left(\omega\left(x_{n}\right)+\left(t-x_{n}\right) \omega^{\prime}\left(x_{n}\right)\right)-\sum_{i=1}^{n-2} \omega\left(z_{i+1}\right) \\
& \geq(n-1) \omega(t)-\sum_{i=1}^{n-2} \omega\left(z_{i+1}\right) \\
& =\left((n-1)-\sum_{i=1}^{n-2} \frac{\omega\left(z_{i+1}\right)}{\omega(t)}\right) \omega(t) \\
& \geq(n-2) \omega(t),
\end{aligned}
$$

since by (4.4)

$$
\sum_{i=1}^{n-2} \frac{\omega\left(z_{i+1}\right)}{\omega(t)} \leq \sum_{i=1}^{n-2} 2^{i+1-n}=1-2^{2-n}
$$

If $y_{n} \leq t<x_{n+1}$ then $\omega(t)-\omega\left(z_{n}\right) \geq 0$, since $\omega$ is increasing and $z_{n} \leq y_{n}$, and thus

$$
\tilde{\omega}(t)=n \omega(t)-\sum_{i=1}^{n-1} \omega\left(z_{i+1}\right) \geq(n-1) \omega(t)-\sum_{i=1}^{n-2} \omega\left(z_{i+1}\right) \geq(n-2) \omega(x)
$$

as in the previous case. Hence (4.8) is proved.

Note that (4.8) shows $\omega(t)=o(\tilde{\omega}(t))$ as $t \rightarrow \infty$; in particular, $\log (t)=o(\tilde{\omega}(t))$ as $t \rightarrow \infty$, since $\omega$ has this property.

On the other hand we have

$$
\tilde{\omega}(t) \leq n \omega(t), \quad \text { if } t \in\left[x_{n}, x_{n+1}\right) \text { and } n \geq 2 .
$$

This is clear by definition if $y_{n} \leq t<x_{n+1}$. On the interval $\left[x_{n}, y_{n}\right]$ the graph of $\tilde{\omega}(x)$ is the line segment with endpoints $\left(x_{n}, \tilde{\omega}\left(x_{n}\right)\right)$ and $\left(y_{n}, \tilde{\omega}\left(y_{n}\right)\right)$. Since $\tilde{\omega}\left(x_{n}\right)=$ $(n-1) \omega\left(x_{n}\right)-\sum_{i=1}^{n-2} \omega\left(z_{i+1}\right) \leq(n-1) \omega\left(x_{n}\right) \leq n \omega\left(x_{n}\right)$ and $\tilde{\omega}\left(y_{n}\right)=n \omega\left(y_{n}\right)-$ $\sum_{i=1}^{n-1} \omega\left(z_{i+1}\right) \leq n \omega\left(y_{n}\right)$ and $n \omega$ is a concave function, we have $\tilde{\omega}(t) \leq n \omega(t)$ for all $t \in\left[x_{n}, y_{n}\right]$ as well. Thus (4.9) is shown.

Definition of $\tilde{\sigma}$. We define $\tilde{\sigma}$ by

$$
\tilde{\sigma}(t):=n \sigma(t)-\sum_{i=1}^{n} \sigma\left(x_{i}\right), \quad \text { for } t \in\left[x_{n}, x_{n+1}\right) .
$$


Then $\tilde{\sigma}$ is a continuous increasing functions such that $t \mapsto \tilde{\sigma}\left(e^{t}\right)$ is convex. We have

$$
\tilde{\sigma}(t) \geq(n-2) \sigma(t), \quad \text { if } t \in\left[x_{n}, x_{n+1}\right) \text { and } n \geq 2 .
$$

In fact, by (4.5)

$$
\tilde{\sigma}(t)=\left(n-\sum_{i=1}^{n} \frac{\sigma\left(x_{i}\right)}{\sigma(t)}\right) \sigma(t) \geq\left(n-\sum_{i=1}^{n} 2^{i-n}\right) \sigma(t) \geq(n-2) \sigma(t) .
$$

This shows $\sigma(t)=o(\tilde{\sigma}(t))$ as $t \rightarrow \infty$; in particular, $\log (t)=o(\tilde{\sigma}(t))$ as $t \rightarrow \infty$.

By definition we have

$$
\tilde{\sigma}(t) \leq n \sigma(t), \quad \text { if } t \in\left[x_{n}, x_{n+1}\right) \text { and } n \geq 2 .
$$

Furthermore, by (4.3),

$$
\tilde{\sigma}(t) \leq \frac{1}{n} f(t), \quad \text { for all } t \geq x_{n},
$$

and hence $\tilde{\sigma}(t)=o(f(t))$ as $t \rightarrow \infty$, as well as

$$
\tilde{\sigma}(t) \leq \frac{1}{n} t, \quad \text { for all } t \geq x_{n}
$$

which shows $\tilde{\sigma}(t)=o(t)$ as $t \rightarrow \infty$.

The pair $(\tilde{\omega}, \tilde{\sigma})$ is $r$-strong for some $r \in(0,1)$. Let $N \in \mathbb{N}_{>2}$ be such that $x_{N} \geq t_{0}$. Let $y \geq x_{N}$. Suppose that $n \in \mathbb{N}_{\geq N}$ is such that $y \in\left[x_{n}, x_{n+1}\right)$. Then $K y \in\left[x_{n}, x_{n+2}\right)$ since $K x_{n+1} \leq x_{n+2}$ by (4.2). By iteration $K^{j} y \in\left[x_{n}, x_{n+j+1}\right]$. Then

$$
\begin{aligned}
& \tilde{\omega}\left(K^{j} y\right) \stackrel{(4.9)}{\leq}(n+j+1) \omega\left(K^{j} y\right) \\
& \stackrel{(4.10)}{\leq} \frac{n+j+1}{n-2} C H^{j} \tilde{\sigma}(y) \leq D j H^{j} \tilde{\sigma}(y)
\end{aligned}
$$

for all $y \geq x_{N}$ and $j \geq 1$ and a constant $D$. Choose $\tilde{H} \in(H, K)$ and $\tilde{N} \in \mathbb{N}$ such that

$$
\frac{\log j}{j} \leq \log \tilde{H}-\log H \quad \text { for all } j \geq \tilde{N}
$$

Then $j H^{j} \leq \tilde{N} \tilde{H}^{j}$ for all $j$. It follows that $\tilde{\omega}$ and $\tilde{\sigma}$ fulfill (3.2) and thus $(\tilde{\omega}, \tilde{\sigma})$ is $r$-strong for some $r \in(0,1)$, by Proposition 7 ,

$\tilde{\omega}$ and $\tilde{\sigma}$ are weight functions. It remains to show $\tilde{\omega}(2 t)=O(\tilde{\omega}(t))$ and $\tilde{\sigma}(2 t)=$ $O(\tilde{\sigma}(t))$ as $t \rightarrow \infty$. For $\tilde{\omega}$ this follows from the concavity, but it is also a consequence of the following argument which we present for $\tilde{\sigma}$. Since $\sigma(2 t)=O(\sigma(t))$ as $t \rightarrow \infty$, there is $N \in \mathbb{N}_{>2}$ and $C \geq 1$ such that

$$
\sigma(2 t) \leq C \sigma(t), \quad \text { for all } t \geq x_{N} .
$$

Fix $t \geq x_{N}$. There is $n \in \mathbb{N}_{\geq N}$ such that $t \in\left[x_{n}, x_{n+1}\right)$ and consequently $2 t \in$ $\left[x_{n}, x_{n+2}\right)$, by (4.2). Then

$$
\tilde{\sigma}(2 t) \stackrel{\text { (4.11) }}{\leq}(n+2) \sigma(2 t) \stackrel{\text { (4.12) }}{\leq}(n+2) C \sigma(t) \stackrel{\text { (4.10) }}{\leq} \frac{n+2}{n-2} C \tilde{\sigma}(t) \leq C^{\prime} \tilde{\sigma}(t) .
$$

Thus $\tilde{\sigma}(2 t)=O(\tilde{\sigma}(t))$ as $t \rightarrow \infty$. The proof is complete. 
Remark 14. Note that $\omega$ and $\tilde{\omega}$ are non-quasianalytic which follows from Re-

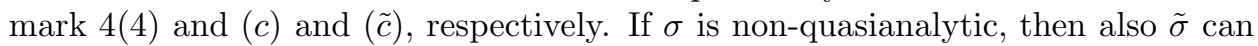
be chosen non-quasianalytic. It suffices to demand

$$
\int_{x_{n}}^{\infty} \frac{\sigma(t)}{1+t^{2}} d t \leq \frac{1}{n^{3}}
$$

in the construction of $\left(x_{n}\right)$. Then

$$
\int_{x_{2}}^{\infty} \frac{\tilde{\sigma}(t)}{1+t^{2}} d t=\sum_{n=2}^{\infty} \int_{x_{n}}^{x_{n+1}} \frac{\tilde{\sigma}(t)}{1+t^{2}} d t \stackrel{(4.11)}{\leq} \sum_{n=2}^{\infty} \int_{x_{n}}^{x_{n+1}} \frac{n \sigma(t)}{1+t^{2}} d t \leq \sum_{n=2}^{\infty} \frac{1}{n^{2}}<\infty .
$$

\section{Proof of Theorem 2}

The proof is based on Lemma 13 and a well-known reduction scheme to the Roumieu case, i.e., Theorem 10. We follow closely the arguments of [4, Theorem $4.5]$ and need two additional lemmas.

Lemma 15 ([6, p.210]). Each weight function $\omega$ is equivalent to a weight function $\bar{\omega}$ of class $C^{1}$. If $\bar{\varphi}=\bar{\omega} \circ \exp$, then $\bar{\varphi}^{\prime}(t) \rightarrow \infty$ as $t \rightarrow \infty$.

Proof. The existence of $\bar{\omega}$ was proved in [6, p.210]. Since $\bar{\omega}$ is a weight function, $\bar{\varphi}=\bar{\omega} \circ \exp$ is convex, $\bar{\varphi}(0)=0$, and $\bar{\varphi}(t) / t \rightarrow \infty$ as $t \rightarrow \infty$. Convexity implies $\bar{\varphi}(t) / t \leq \bar{\varphi}^{\prime}(t)$ for all $t>0$ and hence the statement follows.

Lemma 16 (4, Lemma 4.3]). Let $\left(C_{j}\right)_{j}$ be a positive sequence and let $\left(\psi_{j}\right)_{j}$ be a sequence of differentiable functions on $[0, \infty)$ such that for all $j$ the following conditions are satisfied:

(1) $\psi_{j}$ is convex, increasing, and $\psi_{j}(0)=0$.

(2) $\psi_{j}^{\prime}(t)>\psi_{j+1}^{\prime}(t)$ for all $t>0$.

(3) $\lim _{t \rightarrow \infty}\left(\psi_{j}(t)-\psi_{j+1}(t)\right)=\infty$.

(4) $\lim _{t \rightarrow \infty} \psi_{j}^{\prime}(t)=\infty$.

Then there exist a positive sequence $\left(D_{j}\right)_{j}$ and a convex function $h:[0, \infty) \rightarrow[0, \infty)$ such that

$$
\inf _{j \geq 1}\left(\psi_{j}(t)+C_{j}\right) \leq h(t) \leq \inf _{j \geq 1}\left(\psi_{j}(t)+D_{j}\right) \quad \text { for all } t>0 .
$$

Proof of Theorem Q By Remark 4(2) it suffices to show the extension result for compact sets. So let $K \subseteq \mathbb{R}^{n}$ be a non-empty compact set and fix a Whitney ultrajet $F=\left(F^{\alpha}\right) \in \mathcal{E}^{(\sigma)}(K)$. Consider the sequences

$$
a_{k}:=\sup _{|\alpha|=k} \sup _{x \in E}\left|F^{\alpha}(x)\right|
$$

and

$$
b_{k+1}:=\sup _{|\alpha| \leq k} \sup _{\substack{x, y \in K \\ x \neq y}}\left|\left(R_{x}^{k} F\right)^{\alpha}(y)\right| \frac{(k+1-|\alpha|) !}{|x-y|^{k+1-|\alpha|}}, \quad b_{0}:=0 .
$$

Since $F$ is of class $\mathcal{E}^{(\sigma)}$ and since the Young conjugate $\psi^{*}$ of $\psi: t \mapsto \sigma\left(e^{t}\right)$ is increasing, for each $j \in \mathbb{N}_{\geq 1}$ there exists $C_{j}>1$ such that

$$
\max \left\{a_{k}, b_{k}\right\} \leq C_{j} \exp \left(j \psi^{*}(k / j)\right) \quad \text { for all } k \in \mathbb{N} .
$$

Define the function $g:[0, \infty) \rightarrow \mathbb{R}$ by setting

$$
g(t):=\log \max \left\{a_{k}, b_{k}, 1\right\}, \quad \text { for } k \leq t<k+1 .
$$


Then for each $j \in \mathbb{N}_{\geq 1}$ there exists $C_{j}>0$ such that

$$
g(t) \leq j \psi^{*}(t / j)+C_{j} \quad \text { for all } t \geq 0 .
$$

Let $\psi_{j}(t):=j \psi^{*}(t / j)$. We may assume without loss of generality that $\psi$ is of class $C^{1}$ and $\psi^{\prime}(t) \rightarrow \infty$ as $t \rightarrow \infty$ (by Lemma 15), hence the Young conjugate $\psi^{*}$ is differentiable and satisfies $\left(\psi^{*}\right)^{\prime}=\left(\psi^{\prime}\right)^{-1}$. By Lemma 16 there exist a convex function $h:[0, \infty) \rightarrow[0, \infty)$ and a positive sequence $\left(D_{j}\right)$ such that

$$
g \leq h \leq \inf _{j \geq 1}\left(\psi_{j}+D_{j}\right) .
$$

Then, for each $j \geq 1$ and each $t>0$,

$$
h^{*}(t)=\sup _{s \geq 0}(s t-h(s)) \geq j \sup _{s \geq 0}\left(s t / j-\psi^{*}(s / j)\right)-D_{j}=j \psi(t)-D_{j} .
$$

The function $f(t):=h^{*}(\max \{0, \log (t)\})$ hence satisfies

$$
\sigma(t)=\psi(\log (t)) \leq \frac{1}{j} f(t)+\frac{D_{j}}{j}
$$

for all $j \geq 1$ and all $t \geq 1$. Thus $\sigma(t)=o(f(t))$ as $t \rightarrow \infty$.

By Lemma 13, there exist weight functions $\tilde{\omega}$ and $\tilde{\sigma}$ with the properties $(\tilde{a})(\tilde{c})$ and such that

$$
\omega(t)=o(\tilde{\omega}(t)), \quad \sigma(t)=o(\tilde{\sigma}(t)), \quad \tilde{\sigma}(t)=o(f(t)) \quad \text { as } t \rightarrow \infty .
$$

By Lemma 6 , the pair $(\tilde{\omega}, \tilde{\sigma})$ is strong and consequently satisfies the assumptions of Theorem 1 .

By (5.1), there is a constant $B>0$ such hat $\tilde{\sigma} \leq f+B$. Then we have

$$
\tilde{\psi}(t):=\tilde{\sigma}\left(e^{t}\right) \leq f\left(e^{t}\right)+B=h^{*}(t)+B \quad \text { for all } t \geq 0
$$

and furthermore (since $h$ is convex)

$$
g \leq h=h^{* *} \leq \tilde{\psi}^{*}+B .
$$

This shows that the Whitney ultrajet $F$ belongs to $\mathcal{E}^{\{\tilde{\sigma}\}}(K)$. Then Theorem 1 implies that $F \in j_{K}^{\infty} \mathcal{E}^{\{\tilde{\omega}\}}\left(\mathbb{R}^{n}\right)$. Since, by (5.1), $\omega(t)=o(\tilde{\omega}(t))$ as $t \rightarrow \infty$ and hence $\mathcal{E}^{\{\tilde{\omega}\}}\left(\mathbb{R}^{n}\right) \subseteq \mathcal{E}^{(\omega)}\left(\mathbb{R}^{n}\right)$, Theorem 2 is proved.

\section{EXTENSION OPERATOR}

This section is dedicated to the proof of Theorem 5. It is based on the rather explicit extension procedure available in the Roumieu case; see [23, 24].

Let us recall that due to [20, Theorem 5.14] we have

$$
\mathcal{E}^{(\omega)}\left(\mathbb{R}^{n}\right)=\operatorname{proj}_{U \Subset \mathbb{R}^{n}} \operatorname{proj}_{x>0} \operatorname{proj}_{a>0} \mathcal{E}_{a}^{W^{x}}(U)
$$

and

$$
\mathcal{E}^{\{\omega\}}\left(\mathbb{R}^{n}\right)=\operatorname{proj}_{U \Subset \mathbb{R}^{n}} \operatorname{ind}_{x>0} \operatorname{ind}_{a>0} \mathcal{E}_{a}^{W^{x}}(U)
$$

as locally convex spaces, where

$$
\mathcal{E}_{a}^{W^{x}}(U)=\left\{f \in C^{\infty}(U):\|f\|_{U, a}^{W^{x}}:=\sup _{x \in U} \sup _{\alpha \in \mathbb{N}^{n}} \frac{\left|f^{(\alpha)}(x)\right|}{a^{|\alpha|} W_{|\alpha|}^{x}}<\infty\right\}
$$

is a Banach space associated with the weight sequence

$$
W_{k}^{x}:=\exp \left(\frac{1}{x} \varphi^{*}(k x)\right), \quad k \in \mathbb{N} .
$$

The family $\mathfrak{W}=\left\{W^{x}\right\}_{x>0}$ is called the weight matrix associated with $\omega$. 
If $K$ is a compact subset of $\mathbb{R}^{n}$ then we similarly have topological isomorphisms of the jet spaces

and

$$
\mathcal{E}^{(\omega)}(K)=\operatorname{proj}_{x>0} \operatorname{proj}_{a>0} \mathcal{E}_{a}^{W^{x}}(K)
$$

$$
\mathcal{E}^{\{\omega\}}(K)=\operatorname{ind}_{x>0} \operatorname{ind}_{a>0} \mathcal{E}_{a}^{W^{x}}(K),
$$

where

$$
\mathcal{E}_{a}^{W^{x}}(K)=\left\{F \in \mathcal{E}(K):\|F\|_{K, a}^{W^{x}}+|F|_{K, a}^{W^{x}}<\infty\right\}
$$

and

$$
|F| K, W_{K}^{x}:=\sup _{\substack{x, y \in K \\ x \neq y}} \sup _{p \in \mathbb{N}|\alpha| \leq p} \sup _{|\alpha|}\left|\left(R_{x}^{p} F\right)^{\alpha}(y)\right| \frac{(p+1-|\alpha|) !}{a^{p+1} W_{p+1}^{x}|x-y|^{p+1-|\alpha|}} .
$$

These results are based on the following observation (see [20, (5.10)]): for any weight function $\omega$ we have

$$
\forall a>0 \exists H \geq 1 \forall x>0 \exists C \geq 1 \forall k \in \mathbb{N}: a^{k} W_{k}^{x} \leq C W_{k}^{H x} .
$$

It follows easily that the inclusions $\mathcal{E}_{a}^{W^{x}}\left(\mathbb{R}^{n}\right) \rightarrow \mathcal{E}^{\{\omega\}}\left(\mathbb{R}^{n}\right)$ and $\mathcal{E}^{(\omega)}(K) \rightarrow \mathcal{E}_{a}^{W^{x}}(K)$ are continuous for all $x, a>0$ (cf. [20, Theorem 5.14.1]).

Proof of Theorem [5. We apply Lemma 13 to $f=\tau$. Then there exist weight functions $\tilde{\omega}$ and $\tilde{\sigma}$ satisfying $[(\tilde{a})](\tilde{c})$ as well as $\omega(t)=o(\tilde{\omega}(t)), \sigma(t)=o(\tilde{\sigma}(t))$, and $\tilde{\sigma}(t)=o(\tau(t))$ as $t \rightarrow \infty$.

Let $K$ be a compact subset of $\mathbb{R}^{n}$. Now $\mathcal{E}^{\{\tau\}}(K)$ is continuously included in $\mathcal{E}^{(\tilde{\sigma})}(K)$ thanks to $\tilde{\sigma}(t)=o(\tau(t))$ as $t \rightarrow \infty$, and $\mathcal{E}^{\{\tilde{\omega}\}}\left(\mathbb{R}^{n}\right)$ is continuously included in $\mathcal{E}^{(\omega)}\left(\mathbb{R}^{n}\right)$ since $\omega(t)=o(\tilde{\omega}(t))$ as $t \rightarrow \infty$.

Let $\tilde{\mathfrak{S}}=\{\tilde{S}\}$ be the weight matrix associated with $\tilde{\sigma}$ and $\tilde{\mathfrak{W}}=\{\tilde{W}\}$ the one associated with $\tilde{\omega}$. It was proved in [23, Remark 5.6], 24] that for each $\tilde{S} \in \tilde{\mathfrak{S}}$ and each $a>0$ there exist $\tilde{W} \in \tilde{\mathfrak{W}}, b>0$, and a continuous linear extension operator $\mathcal{E}_{a}^{\tilde{S}}(K) \rightarrow \mathcal{E}_{b}^{\tilde{W}}\left(\mathbb{R}^{n}\right)$. Since also the natural inclusions $\mathcal{E}^{(\tilde{\sigma})}(K) \rightarrow \mathcal{E}_{a}^{\tilde{S}}(K)$ and $\mathcal{E}_{b}^{\tilde{W}}\left(\mathbb{R}^{n}\right) \rightarrow \mathcal{E}^{\{\tilde{\omega}\}}\left(\mathbb{R}^{n}\right)$ are continuous, there exists a continuous linear extension operator $\mathcal{E}^{\{\tau\}}(K) \rightarrow \mathcal{E}^{(\omega)}\left(\mathbb{R}^{n}\right)$.

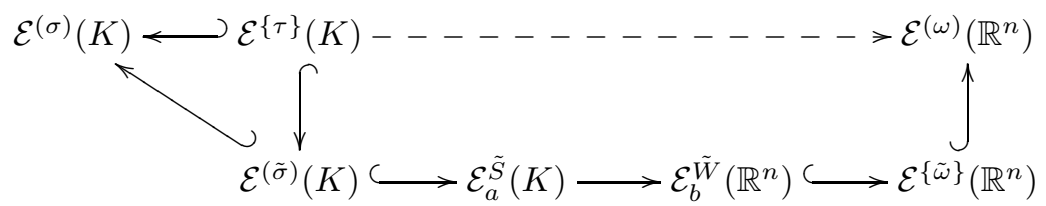

If $A$ is a closed subset of $\mathbb{R}^{n}$, then we may use a suitable partition of unity as in Remark 4(2) in order to obtain the required extension operator.

\section{REFERENCES}

[1] A. V. Abanin, On Whitney's extension theorem for spaces of ultradifferentiable functions, Math. Ann. 320 (2001), no. 1, 115-126.

[2] A. Beurling, Quasi-analyticity and general distributions, Lecture notes, AMS Summer Institute, Stanford, 1961.

[3] G. Björck, Linear partial differential operators and generalized distributions, Ark. Mat. 6 (1966), 351-407.

[4] J. Bonet, R. W. Braun, R. Meise, and B. A. Taylor, Whitney's extension theorem for nonquasianalytic classes of ultradifferentiable functions, Studia Math. 99 (1991), no. 2, 155-184. 
[5] J. Bonet, R. Meise, and B. A. Taylor, On the range of the Borel map for classes of nonquasianalytic functions, Progress in functional analysis (Peñiscola, 1990), North-Holland Math. Stud., vol. 170, North-Holland, Amsterdam, 1992, pp. 97-111.

[6] R. W. Braun, R. Meise, and B. A. Taylor, Ultradifferentiable functions and Fourier analysis, Results Math. 17 (1990), no. 3-4, 206-237.

[7] J. Chaumat and A.-M. Chollet, Surjectivité de l'application restriction à un compact dans des classes de fonctions ultradifférentiables, Math. Ann. 298 (1994), no. 1, 7-40.

[8] L. Ehrenpreis, Fourier analysis in several complex variables, Pure and Applied Mathematics, Vol. XVII, Wiley-Interscience Publishers A Division of John Wiley \& Sons, New York-LondonSydney, 1970.

[9] U. Franken, Continuous linear extension of ultradifferentiable functions of Beurling type, Math. Nachr. 164 (1993), 119-139.

[10] J. Jiménez-Garrido, J. Sanz, and G. Schindl, Indices of o-regular variation for weight functions and weight sequences, Revista de la Real Academia de Ciencias Exactas, Físicas y Naturales. Serie A. Matemáticas 113 (2019), no. 4, 3659-3697.

[11] Sectorial extensions, via laplace transforms, in ultraholomorphic classes defined by weight functions, Results in Mathematics 74 (2019), no. 1.

[12] _ Ultraholomorphic extension theorems in the mixed setting, Banach Journal of Mathematical Analysis 14 (2020), no. 4, 1630-1669.

[13] M. Langenbruch, Extension of ultradifferentiable functions of Roumieu type, Arch. Math. (Basel) 51 (1988), no. 4, 353-362.

[14] Extension of ultradifferentiable functions, Manuscripta Math. 83 (1994), no. 2, 123143.

[15] — A general approximation theorem of Whitney type, RACSAM Rev. R. Acad. Cienc. Exactas Fís. Nat. Ser. A Mat. 97 (2003), no. 2, 287-303.

[16] R. Meise and B. A. Taylor, Whitney's extension theorem for ultradifferentiable functions of Beurling type, Ark. Mat. 26 (1988), no. 2, 265-287.

[17] _ Linear extension operators for ultradifferentiable functions of Beurling type on compact sets, Amer. J. Math. 111 (1989), no. 2, 309-337.

[18] H.-J. Petzsche, On E. Borel's theorem, Math. Ann. 282 (1988), no. 2, 299-313.

[19] A. Rainer and G. Schindl, Composition in ultradifferentiable classes, Studia Math. 224 (2014), no. 2, 97-131.

[20] — Composition in ultradifferentiable classes, Studia Math. 224 (2014), no. 2, 97-131.

[21] Extension of Whitney jets of controlled growth, Math. Nachr. 290 (2017), no. 14-15, 2356-2374, doi:10.1002/mana.201600321.

[22] _ On the Borel mapping in the quasianalytic setting, Math. Scand. 121 (2017), 293310.

[23] On the extension of Whitney ultrajets, Studia Math. 245 (2019), no. 3, 255-287.

[24] - On the extension of Whitney ultrajets, II, Studia Math. 250 (2020), no. 3, 283-295.

[25] J. Schmets and M. Valdivia, Analytic extension of ultradifferentiable Whitney jets, Collect. Math. 50 (1999), no. 1, 73-94.

[26] - On certain extension theorems in the mixed Borel setting, J. Math. Anal. Appl. 297 (2004), no. 2, 384-403, Special issue dedicated to John Horváth.

[27] V. Thilliez, Division by flat ultradifferentiable functions and sectorial extensions, Results Math. 44 (2003), no. 1-2, 169-188.

[28] H. Whitney, Analytic extensions of differentiable functions defined in closed sets, Trans. Amer. Math. Soc. 36 (1934), no. 1, 63-89.

A. Rainer: Fakultät Für Mathematik, Universität Wien, Oskar-MorgensternPlatz 1, A-1090 Wien, Austria

Email address: armin.rainer@univie.ac.at 